- Case Report

\title{
Chronic Large Non Healing Ulcer: Non- Tuberculous Mycobacterial Infection of the Laryngopharynx
}

\author{
Nurfarissa Hussin, Marina Mat Baki*, Abdullah Sani \\ Department of Otorhinolaryngology, Universiti Kebangsaan Malaysia Medical Centre, Kuala Lumpur, Malaysia
}

We describe a case of non-tuberculous mycobacterial infection of the larynx in a previously well, immunocompetent young woman. Laryngeal mycobacterial infection is rare and currently accounts for less than $1 \%$ of all cases. A diagnostic dilemma often occurs because it may mimic laryngeal carcinoma, chronic laryngitis, or laryngeal candidiasis. This case highlights the importance of considering non-tuberculous mycobacterial infection in the differential diagnosis of laryngeal lesions.

Keywords: Nontuberculous Mycobacteria; Larynx; Pharynx; Laryngopharynx 


\section{INTRODUCTION}

Non-tuberculous mycobacterial infection has been described in the medical literature as early as the mid-1950s. ${ }^{1,2)}$ With the development of and advances in the field of mycobacteriology over the past 20 years, other mycobacteria can be distinguished from Mycobacterium tuberculosis and antimicrobial susceptibility testing of mycobacteria can be performed.

Non-tuberculous mycobacteria (NTM) are classified based on their growth rates. Rapidly growing NTM is categorized into pigmented and non-pigmented species. Examples of pigmented species are M. phlei, M. aurum, and M. flavescens, but they are rarely associated with clinical diseases. The non-pigmented NTM species are M. fortuitum complex and the M. chelonae/abscessus group. ${ }^{3)}$

Here, a case of non-tuberculous mycobacterial infection of the laryngopharynx with an unusual presentation of a painful large ulcer is discussed.

\section{CASE REPORT}

In December 2009, a previously well 30-year-old pharmacist presented with a chronic recurrent sore throat since 1 year. The symptom started during her last pregnancy and was preceded by multiple oral ulcers. The sore throat was mainly on the right side and was associated with odynophagia and a foreign body sensation. She had no fever or other constitutional symptoms. Clinically, there was no stridor, hoarseness, or lymphadenopathy.

On examination of the larynx using a rigid 70-degree scope, there was an ulcer visible over the right hypopharynx and on the lingual surface of the epiglottis (Figure 1). The aryepiglottic fold was erythematous and edematous. The patient underwent direct laryngoscopy and

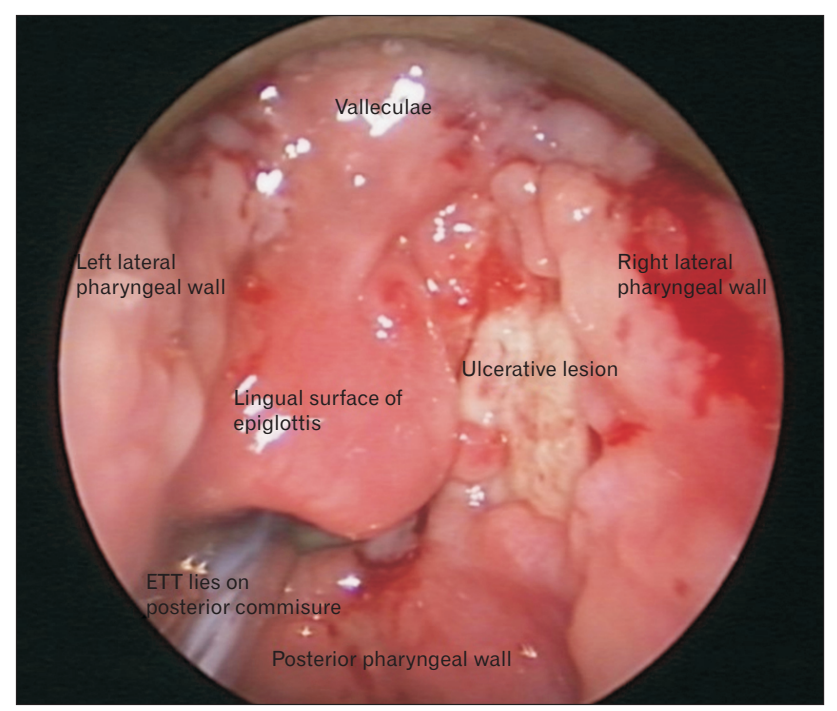

Figure 1. This figure shows an endoscopic view of the ulcerative lesion seen over the right hypopharynx and lingual surface of the epiglottis. The aryepiglottic fold is erythematous and edematous. ETT, endotracheal tube. laser excision of the ulcer.

Histopathological examination revealed a benign ulcer with evidence of granulation without granuloma or neoplastic changes. ZiehlNeelsen and fungal staining was negative.

The patient was then well until 3 months post operation when the symptoms recurred. On examination, there was an ulcer at the left hypopharynx, extending to the lateral wall, crossing the midline, and involving the lingual surface of the epiglottis (Figure 2). The vocal folds appeared normal and mobile bilaterally. She again underwent endolaryngeal microsurgery and excision of the ulcer. Histopathological examination revealed similar findings.

Subsequently, the patient was investigated for evidence of tuberculosis infection, which included a chest X-ray scan, Mantoux test, erythrocyte sedimentation rate assay, C-reactive protein assay, and acid-fast bacilli culture of sputum, all of which showed negative results. Autoimmune studies and infectious disease investigations including human immunodeficiency virus (HIV), Venereal Disease Research Laboratory, and hepatitis $\mathrm{B}$ and $\mathrm{C}$ were also all negative. Finally, tuberculosis polymerase chain reaction (PCR) assay for the tissue biopsy taken at the hypopharynx was positive for a mycobacterium other than $M$. tuberculosis.

The patient was then treated with oral ciprofloxacin, clarithromycin, and doxycycline for atypical mycobacterium for 6 weeks. Unfortunately, 2 months after the diagnosis and completion of her course of antibiotics, symptoms recurred, and she developed a sore throat associated with productive cough, dysphagia, and odynophagia. She did not experience any difficulty in breathing.

Laryngeal examination revealed a hypopharyngeal wall with a fibrous border and stenosis over the anterior part of the hypopharynx (Figure 3). The epiglottis was retracted and the pyriform fossa and vocal folds were unable to be visualized. Tracheostomy and endolaryngeal microsurgery with laser excision of the fibrous band and application of mitomycin $\mathrm{C}$ was then performed under general anesthesia.



Figure 2. This figure shows an endoscopic view of a large ulcer seen at the left hypopharynx, extending to the lateral wall, crossing the midline, and involving the lingual surface of the epiglottis. 


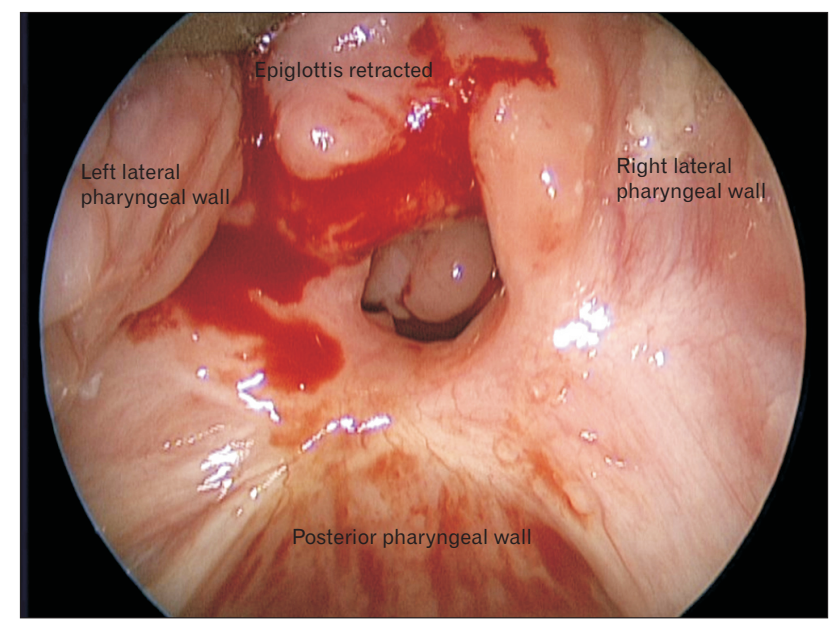

Figure 3. This figure shows an endoscopic view of the larynx that depicts the hypopharyngeal wall with a fibrous border and stenosis over the anterior part of hypopharynx. The epiglottis was retracted and the pyriform fossa and also the vocal folds are unable to be visualized.

Postoperatively the patient was well, had no difficulty in breathing, and the tracheostomy was decannulated 4 days post operation.

The patient was seen in the clinic 1 month after the procedure and she was well and asymptomatic. During subsequent clinic visits, the stenotic region had not worsened and the airway remained good and patent. She has remained well so far.

\section{DISCUSSION}

Non-tuberculous mycobacterial infection was first described in the medical literature as early as the mid-1950s. ${ }^{1,2)}$ NTM are classified based on their growth rates. Rapidly growing NTM is categorized into pigmented and non-pigmented species. ${ }^{3)}$ NTM are also called atypical mycobacteria or mycobacteria other than tuberculosis. It is a group of opportunistic bacterial pathogens numbering more than 120 species. Infection is acquired from environmental reservoirs and usually manifests as pulmonary disease, although lymphadenitis, soft tissue infection, and bacteremia may also occur. ${ }^{4)}$ They are divided into two main groups: (1) the slow grower; and (2) the rapid grower, according to their rate of growth in subculture. Most of the slow growers are able to cause human diseases, the most common being two members of the M. avium complex (MAC), i.e., M. avium and M. avium intracellulare. NTM is ubiquitous in the environment but $M$. tuberculosis infection has always overshadowed the true prevalence of NTM. ${ }^{4)}$ Diseases caused by NTM are not generally notifiable and, as a result, their epidemiology is not well described. ${ }^{5}$ Although the exact magnitude of NTM is difficult to predict because a culture is not a routine part of diagnosing tuberculosis, it is thought to vary from $1 \%$ to $28 \%$. $^{4)}$

NTM are environmental bacteria that humans encounter on a daily basis; thus, diagnosing pulmonary NTM disease is not straightforward because a single positive culture from nonsterile sources including the respiratory or digestive tract does not indicate infection or disease. The
American Thoracic Society and Infectious Diseases Society of America have issued a set of criteria to differentiate normal NTM isolation from true pulmonary NTM disease, which include clinical, radiological, and microbiological evidence of disease that should be gathered in order to diagnose NTM infection. ${ }^{6}$

Generally, the symptoms of NTM infection are nonspecific. Most patients will present with a chronic cough, with or without sputum production or hemoptysis and fatigue or malaise. Constitutional symptoms such as weight loss, fever, and night sweats are less frequent and occur in $30 \%$ to $50 \%$ of patients. Once constitutional symptoms develop, it is usually a case of advanced disease. ${ }^{6)}$ As for our patient, she presented with a chronic sore throat associated with odynophagia and a foreign body sensation; which is a nonspecific and uncommon symptom of NTM infection.

In extrapulmonary NTM disease, lymphadenitis caused by NTM usually affects lymph nodes at a single site. The submandibular and cervical lymph nodes are most frequently affected, although axillary and inguinal lymphadenitis has been observed. Patients are typically children under the age of 8 years; but it can occur in any age group. This disease is relatively benign, and most patients present with an enlarged lymph node without constitutional symptoms. In more advanced disease, fluctuating masses with violaceous overlying skin are seen. Fine-needle aspirate cytology or excised lymph nodes are the specimen of choice to obtain microbiological evidence of NTM disease.

Our understanding of NTM is largely based on the more common MAC infections. The major clinical settings for MAC disease in HIV negative patients are upper lobe cavitating pulmonary disease in middle-aged men with heavy alcohol and tobacco consumption and elderly non-smokers who have evidence of multiple nodular pulmonary infiltrates. ${ }^{7)}$ Other less-frequent clinical settings include disseminated disease in patients with advanced acquired immune deficiency syndrome and rarely in non-HIV patients who have previously been treated with steroids. ${ }^{8)}$

According to a statement by the American Thoracic Society and the Infectious Diseases Society of America in 2007, medical treatment of MAC pulmonary disease in HIV negative patients with antituberculosis medications has yielded inconsistent results. The major advancement in the treatment of MAC was the introduction of the newer macrolides. This patient was given a course of antibiotics, including clarithromycin.

In the case we presented, an initial diagnosis of laryngopharynx NTM infection was made on the basis of a positive atypical tuberculosis PCR. To the best of our knowledge, there have been no published cases of NTM involving the hypopharyngeal structures. Diagnosis was delayed due to a low suspicion index of atypical mycobacterial infection and other investigations, including tuberculosis culture, were negative after 6 weeks. Mycobacterial smear and culture are difficult because in these locations the concentration of the bacilli is low. An accurate diagnosis can be made by PCR from oral samples with a high sensitivity ( $89 \%$ to $100 \%){ }^{9)}$ 
In conclusion, non-tuberculous mycobacterial infection should be considered in the differential diagnosis of a patient presenting with a chronic painful ulcer especially when all tuberculosis investigations reveal negative results. A PCR test is recommended when non-tuberculous or tuberculous mycobacteria infection is highly suspected.

\section{CONFLICT OF INTEREST}

No potential conflict of interest relevant to this article was reported.

\section{REFERENCES}

1. Masson AM, Prissick FH. Cervical lymphadenitis in children caused by chromogenic Mycobacteria. Can Med Assoc J 1956;75:798-803.

2. Weed LA, Keith HM, Needham GM. Nontuberculous acid-fast cervical adenitis in children. Proc Staff Meet Mayo Clin 1956;31:259-63.

3. McEwan JA, Mohsen AH, Schmid ML, McKendrick MW. A hoarse voice: atypical mycobacterial infection of the larynx. J Laryngol Otol
2001;115:920-2.

4. Russell CD, Claxton P, Doig C, Seagar AL, Rayner A, Laurenson IF. Non-tuberculous mycobacteria: a retrospective review of Scottish isolates from 2000 to 2010. Thorax 2014;69:593-5.

5. Van Ingen J, Bendien SA, de Lange WC, Hoefsloot W, Dekhuijzen PN, Boeree MJ, et al. Clinical relevance of non-tuberculous mycobacteria isolated in the Nijmegen-Arnhem region, The Netherlands. Thorax 2009;64:502-6.

6. Mandell GL, Bennett JE, Dolin R. Principles and practice of infectious diseases. 5th ed. Philadelphia (PA): Churchill Livingstone; 2000.

7. Shin JE, Nam SY, Yoo SJ, Kim SY. Changing trends in clinical manifestations of laryngeal tuberculosis. Laryngoscope 2000;110:1950-3.

8. Falkinham JO. Impact of human activities on the ecology of nontuberculous mycobacteria. Future Microbiol 2010;5:951-60.

9. Griffith DE, Aksamit T, Brown-Elliott BA, Catanzaro A, Daley C, Gordin F, et al. An official ATS/IDSA statement: diagnosis, treatment, and prevention of nontuberculous mycobacterial diseases. Am J Respir Crit Care Med 2007;175:367-416. 